\title{
Figure of Merit For Muon Cooling - An Algorithm for Particle Counting in Coupled Phase Planes
}

\author{
E.B. Holzer
}

\begin{abstract}
A method to count particles in hyper-ellipsoidal phase space volumes was developed to be used as figure of merit for a muon cooling experiment and as a measure for the cooling performance of a neutrino factory cooling channel. The cooling rate in four and six dimensions can be measured in the presence of coupling between phase space planes. The algorithm was demonstrated on simulations of the cooling experiment.
\end{abstract}




\title{
Figure of Merit for Muon Cooling - An Algorithm for Particle Counting in Coupled Phase Planes
}

\author{
Eva Barbara Holzer \\ AB Division, CERN, CH-1211 Genève 23, Switzerland
}

\begin{abstract}
A method to count particles in hyper-ellipsoidal phase space volumes was developed to be used as figure of merit for a muon cooling experiment and as a measure for the cooling performance of a neutrino factory cooling channel. The cooling rate in four and six dimensions can be measured in the presence of coupling between phase space planes. The algorithm was demonstrated on simulations of the cooling experiment.
\end{abstract}

Key words: ionization cooling, figure of merit, hyper-ellipsoids, coupling PACS:

\section{Introduction}

In the design scenarios of a neutrino factory [1-3] intense beams of electron and muon neutrinos are produced by high energy muons decaying in the long straight sections of a muon decay ring. These muons are decay products of pions which are produced by high intensity proton beams impinging on a target. Ionization cooling of the muons is proposed to increase the phase space density and hence the flux of neutrinos emitted towards the far distance detectors.

In the cooling channel of a Neutrino Factory the aim is to increase the number of muons in the (limiting) acceptance of the following accelerators and the decay ring. This acceptance is defined independently in the three planes $(x, y$ and longitudinal). The CERN and US baseline scenarios use solenoidal focusing from the pion production target to the muon cooling channel $[1,2]$. Coupling leads to correlations between the planes which have to be minimised at the point of transition from solenoidal focusing to quadrupole focusing. To achieve simultaneous cooling in the longitudinal and transverse planes strong coupling 
(emittance exchange between longitudinal and transverse phase space) is actually intended. Depending on the optics, areas of important correlations between the planes will exist along the cooling channel. To design and optimise a cooling channel the cooling performance needs to be followed all along the channel including the areas of high correlations. The aim of a muon cooling experiment is to confirm the feasibility of muon ionization cooling, to quantify the achievable cooling performance and to explore the parameter space [5]. In the cooling experiment the emittances are measured inside the solenoid field, at a position where inter-plane correlations can be substantial.

Therefore, an algorithm was developed to count particles in arbitrary dimensional hyper-ellipsoidal phase space volumes, to be used as figure of merit for the cooling experiment in the presence of inter-plane correlations and as a measure for the cooling performance of a neutrino factory cooling channel. The new method allows to measure $4 \mathrm{D}$ and $6 \mathrm{D}$ cooling rather than the $2 \mathrm{D}$ projections. In a solenoidal beam transport for transverse cooling mainly correlations between the $x$ and the $y$ plane will develop, and it is sufficient to investigate the $4 \mathrm{D}$ transverse phase space independently. The algorithm in $6 \mathrm{D}$ can be applied to scenarios with emittance exchange between the longitudinal and transverse planes.

In section 2 the standard formula for counting particles in two dimensional phase space ellipses is recapitulated. Thereafter, the generalisation to arbitrary dimensional hyper-ellipsoids is derived. The new algorithm (in 4D) is demonstrated, in section 3, on the $88 \mathrm{MHz}$ and $200 \mathrm{MHz}$ cooling experiment simulations. Some elementary definitions and equations are collected in the appendix.

\section{Counting particles inside a hyper-ellipsoid}

To follow the progress of cooling throughout the channel, the number of muons inside a 2D, 4D or 6D ellipsoidal volume is counted at different positions along the $z$-axis (longitudinal direction). The orientation of the ellipsoid is optimised at each $z$-position to maximise the number of muons inside the volume. In the derivation of the formula the orientation of the ellipsoid is first assumed to be adapted to the muon beam and then generalised to arbitrary orientation. 
In the standard procedure of counting particles inside a 2D ellipse a single particle emittance, $\epsilon_{0}$, is defined for each individual particle:

$$
\epsilon_{0}=\epsilon X_{0}^{\mathrm{T}} \Sigma^{-1} X_{0}
$$

In this paper the emittance, $\epsilon$, is defined as the volume (area) of the ellipsoid (ellipse) defined by the beam sigma matrix (see the appendix for details). $X_{0}$ is the coordinate vector of the particle. $\epsilon_{0}$ is the volume (area) of an ellipsoid (ellipse) on which the particle coordinate vector $X_{0}$ lies and which is similar (same orientation and aspect ratio) to the rms emittance ellipse of the muon beam. For a particle to be inside a certain acceptance its $\epsilon_{0}$ has to be smaller than the $\epsilon_{\text {limit }}$ (area) of the acceptance ellipse:

$$
X_{0}^{\mathrm{T}} \Sigma^{-1} X_{0} \times \epsilon<\epsilon_{\text {limit }}
$$

\section{Counting in a hyper-ellipsoid of arbitrary dimensions}

In the following, the formalism for arbitrary dimensions, $k$, is derived. As in two dimensions the rms emittance is determined from the particle distribution. Then the acceptance is defined as a hyper-ellipsoid which has the same orientation in $k \mathrm{D}$ phase space as the beam particle distribution itself. After having defined the orientation of the acceptance ellipsoid it needs to be checked for each individual particle whether it lies inside the acceptance volume. For this purpose a $k$ dimensional single particle emittance, $\epsilon_{0}$, is associated with the particle. As in 2D, $\epsilon_{0}$ is defined as the volume of a hyper-ellipsoid which is oriented the same way as the acceptance, and on which the particle lies. If the single particle emittance is smaller than the acceptance, the particle falls inside the acceptance.

Expressed in mathematical terms we have a statistical ensemble of particles in $k$ dimensions with its $k \mathrm{D}$ sigma matrix, $\Sigma$, defining its orientation in space. The orientation of the acceptance hyper-ellipsoid is given by the orientation of $\Sigma$. The individual particle to be investigated has the coordinate vector $X_{0}$. Associated with the particle is the matrix $\Sigma_{0}$. It defines a hyper-ellipsoid with the same orientation as the particle distribution and on which the particle lies. The condition of similarity of the hyper-ellipsoids implies that $\Sigma_{0}$ is proportional to $\Sigma$

$$
\Sigma_{0}=\Sigma \Delta
$$

where $\Delta$ is the proportionality factor. The definition of a hyper-ellipsoid which contains the point $X_{0}$ is

$$
X_{0}^{\mathrm{T}} \Sigma_{0}^{-1} X_{0}=1
$$

Therefore

$$
\Delta=X_{0}^{\mathrm{T}} \Sigma^{-1} X_{0} \quad \text { and } \quad \Sigma_{0}=\Sigma X_{0}^{\mathrm{T}} \Sigma^{-1} X_{0}
$$


The single particle emittance, $\epsilon_{0}$, is the volume of the hyper-ellipsoid defined by $\Sigma_{0}$. Therefore (see appendix $(11) ; g_{k}$ is a geometric factor):

$$
\epsilon_{0}=g_{k} \sqrt{\operatorname{det} \Sigma_{0}}=g_{k} \sqrt{\operatorname{det} \Sigma} \Delta^{k / 2}=\epsilon_{\mathrm{rms}} \Delta^{k / 2},
$$

i.e., $\epsilon_{0}$ is $\Delta^{k / 2}$ times the volume of the rms hyper-ellipsoid,

$$
\epsilon_{0}=g_{k} \sqrt{\operatorname{det} \Sigma}\left(X_{0}^{\mathrm{T}} \Sigma^{-1} X_{0}\right)^{k / 2} .
$$

Equation (7) defines a single particle emittance in arbitrary dimensions. For a particle to lie inside the acceptance hyper-ellipsoid it has to fulfil the condition that its emittance $\epsilon_{0}$ is smaller than the limit, $\epsilon_{\text {limit }}$, which is the volume of the acceptance hyper-ellipsoid:

$$
\epsilon_{0}<\epsilon_{\text {limit }}
$$

and finally:

$$
X_{0}^{\mathrm{T}} \Sigma^{-1} X_{0}<\left(\frac{\epsilon_{\text {limit }}}{\epsilon_{\mathrm{rms}}}\right)^{2 / k}
$$

Equation (9) is the criterion for a particle to lie inside an acceptance volume of hyper-ellipsoidal shape in arbitrary dimensions. In the case of two dimensions we recognise the familiar condition of (2). Because of the averaging in the calculation of $\Sigma$ (see appendix (12)) $X_{0}^{\mathrm{T}} \Sigma^{-1} X_{0}$ will in general depend on the coordinate system in which the sigma matrix was calculated. In view of the high momentum spread and divergence of the muons beams, it should be calculated in canonical coordinates.

When the orientation of the acceptance volume needs to be chosen different from the orientation of the rms hyper-ellipsoid, e.g. in the presence of tails in the distribution, the formulas can easily be adapted. The beam sigma matrix in (7) is replaced by a matrix defining an ellipsoid with the desired orientation. The size of the ellipsoid is of no importance, as it cancels out. Condition (8) is then used to determine whether a particle falls inside the acceptance. An iterative procedure can be applied to find the orientation with the highest particle count.

\section{Application Example}

The algorithm has been applied to the simulation of two cooling experiment set-ups [5]. They are based on the $88 \mathrm{MHz}$ CERN cooling channel design [1] and the $200 \mathrm{MHz}$ US study II design [2] respectively. Both use solenoidal beam focusing and consist of an entrance diagnosis section (constant $B_{z}$ ), a liquid hydrogen absorber, a string of RF cavities, a second absorber and the exit diagnosis section. The muon energy is $200 \mathrm{MeV} \pm 30 \mathrm{MeV}$ in both 
simulations. The energy loss and gain, in absorbers and cavities respectively, is $4.4 \%$ higher in the $200 \mathrm{MHz}$ experiment. The average magnetic field at the absorber is $1.95 \mathrm{~T}$ in the $88 \mathrm{MHz}$ setup and $3.04 \mathrm{~T}$ in the $200 \mathrm{MHz}$ case. The cooling efficiency of both systems is comparable. The beam optics of the two experiments is very different. Strong inter-plane correlations build up in the $200 \mathrm{MHz}$ design, while they are absent in the $88 \mathrm{MHz}$ case.
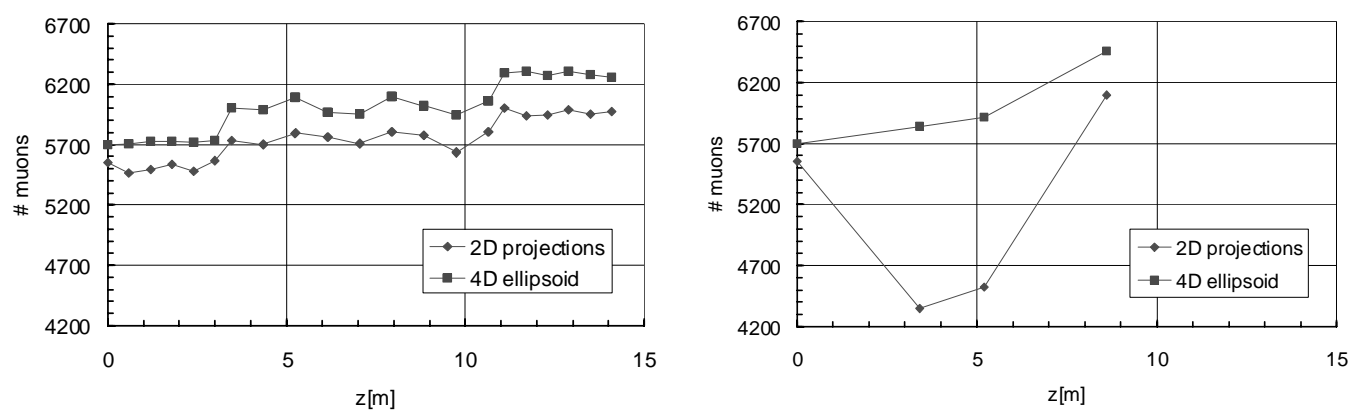

Fig. 1. Number of muons inside $4 \mathrm{D}$ volume of $(0.015 \pi \mathrm{m} \mathrm{rad})^{2}$ along the $88 \mathrm{MHz}$ (left) and $200 \mathrm{MHz}$ (right) cooling experiment. The upper curves correspond to a $4 \mathrm{D}$ ellipsoid, the lower curves to $2 \mathrm{D}$ projections.

Figure 1 shows the number of muons inside a normalised $4 \mathrm{D}$ volume of $(0.015 \pi \mathrm{m} \mathrm{rad})^{2}$ along the $z$-position for the $88 \mathrm{MHz}$ (left side) and the $200 \mathrm{MHz}$ (right side) experiment respectively. The upper curve is the number of muons in a $4 \mathrm{D}$ ellipsoid while the lower one represents the count in $2 \mathrm{D}$ projections. In the $88 \mathrm{MHz}$ setup the correlations between the $x$ and $y$ plane stay below 0.08. In the absence of correlations the $4 \mathrm{D}$ method reproduces the result of the $2 \mathrm{D}$ particle counting. As the 4D ellipsoid looks at a more central core of the distribution the density of muons is higher and the increase in muons is slightly larger than in the 2D projections (9.9\% increase as compared to $7.6 \%$ in the $2 \mathrm{D}$ projections). For the $200 \mathrm{MHz}$ simulation four $z$-positions are shown, the beginning of the experiment, two intermediate positions (where $\epsilon_{x} \times \epsilon_{y}$ peaks) and the end of the experiment. The corresponding inter-plane correlations are 0, 0.7, 0.6 and 0.14 respectively. The number of muons increases by $13.3 \%$ in the $4 \mathrm{D}$ volume and by $9.9 \%$ in the $2 \mathrm{D}$ projections. At the points of high correlations the counting in $2 \mathrm{D}$ projections completely fails to represent the cooling performance and the 4D (or 6D) method becomes necessary to asses it.

\section{Conclusions}

An algorithm was derived to count particles in an acceptance of four and six dimensional ellipsoids. The application to cooling experiment simulations shows that it yields a stable figure of merit in the presence of correlations, where 
the 2D method fails. This is of high importance when assessing the cooling performance of muon ionization cooling channels, where strong coupling is present.

\section{Appendix: Definitions}

The general equation of a hyper-ellipsoid in $k$ dimensions at the origin of the coordinate system is:

$$
u^{\mathrm{T}} \sigma^{-1} u=1 .
$$

$\sigma$ is a $k \times k$ matrix and $u$ is the $k$-dimensional coordinate vector. $\sigma$ is symmetric invertible and all eigenvalues are positive. The volume of the ellipsoid is:

$$
\text { Volume }=g_{k} \sqrt{\operatorname{det} \sigma}, \quad g_{k}=\frac{\pi^{k / 2}}{\Gamma(1+k / 2)}
$$

Hence, $g_{2}=\pi, g_{4}=\frac{\pi^{2}}{2}$ and $g_{6}=\frac{\pi^{3}}{6}$. For a beam of particles (particle coordinate vector $\vec{x}$ ) in $k$ dimensions the sigma matrix is defined as:

$$
\Sigma_{i j}^{k \mathrm{D}}=\left\langle\left(x_{i}-\overline{x_{i}}\right)\left(x_{j}-\overline{x_{j}}\right)\right\rangle
$$

In this note we define the rms emittance in $k$ dimensions as the volume (in $2 \mathrm{D}$ the area) of the hyper-ellipsoid defined by the beam sigma matrix (12):

$$
\epsilon_{\mathrm{rms}}^{k \mathrm{D}} \equiv g_{k} \sqrt{\operatorname{det} \Sigma^{k \mathrm{D}}} \text {. }
$$

The relation between the $2 \mathrm{D}$ rms emittances and the $4 \mathrm{D}$ and $6 \mathrm{D}$ rms emittances for an uncorrelated beam is:

$$
\begin{gathered}
\epsilon_{\mathrm{rms}}^{6 \mathrm{D}}=\frac{\pi^{3}}{6} \sqrt{\operatorname{det} \Sigma_{x}^{2 \mathrm{D}} \cdot \operatorname{det} \Sigma_{y}^{2 \mathrm{D}} \cdot \operatorname{det} \Sigma_{z}^{2 \mathrm{D}}}=\frac{1}{6} \epsilon_{x} \epsilon_{y} \epsilon_{z}, \\
\epsilon_{\mathrm{rms}}^{4 \mathrm{D}}=\frac{1}{2} \epsilon_{x} \epsilon_{y},
\end{gathered}
$$

where $\Sigma_{x}^{2 \mathrm{D}}, \Sigma_{y}^{2 \mathrm{D}}$ and $\Sigma_{z}^{2 \mathrm{D}}$ are the $2 \mathrm{D}$ sigma matrices in the horizontal, vertical and longitudinal plane respectively. $\epsilon_{x}, \epsilon_{y}$ and $\epsilon_{z}$ denote the $2 \mathrm{D}$ rms emittances. The volume of a $6 \mathrm{D}$ (4D) hyper-ellipsoid of an uncorrelated beam is a factor of $1 / 6(1 / 2)$ smaller than the product of the projected $2 \mathrm{D}$ areas.

\section{References}

[1] Prospective study of muon storage rings at CERN, eds. B. Autin, A. Blondel and J. Ellis, CERN yellow report CERN 99-02, ECFA 99-197 (1999). The Study of a 
European Neutrino Factory Complex, ed. P. Gruber, CERN/PS 2002-080(PP) (2002).

[2] A Feasibility Study of a Neutrino Source based on a Muon Storage Ring, eds. N. Holtkamp and D. Finley (2000). Feasibility Study-II of a Muon-Based Neutrino Source, eds. S. Ozaki, R. Palmer, M. Zisman, and J. Gallardo, BNL-52623 (2001).

[3] A Feasibility Study of A Neutrino Factory in Japan, ed. Y. Kuno (2001).

[4] Proposal to the Rutherford Appleton Laboratory: An International Muon Ionization Cooling Experiment (MICE), ed. P. Gruber, (2003). MUCOOL experiment Muon Ionization Cooling R\&D, FNAL Proposal P904. Status of Neutrino Factory and Muon collider Research and Development and Future Plans, M.M. Alsharo'a et al. (2002).

[5] Beam Dynamics Study of a Muon Ionization Cooling Experiment, M. Aleksa et al., CERN-Nufact-Note-108. 